special interest-the South Wales valleys, SheffieldRotherham (Geddes' South Riding), the Lancashire Coalfield (Wigan-St. Helens), and the Potteries. Some of these areas already form more extensive and almost contiguous clusters, as envisaged by Geddes. The whole South Wales area (Geddes' Waleston) has $1 \cdot 1$ million people. Nottingham and Derby are also linked with 850,000 . The Sheffield and Barnsley areas are virtually one. Manchester and Liverpool are interconnected through the Lancashire coalfield area (Geddes' Lancaston). The smaller conurbations $(50,000-250,000), 75$ in number, are treated in two chapters. Portrayals are necessarily brief and encycloprdic.

This book is essentially a descriptive compilation. This is how Freeman chose to write it; but the approach will not advance geography as a research discipline or as a contributor to planning. He does not formulate and systematically develop any geographical concepts with regard to urban growth, an approach that would necessitate a complete reorganization of the book, but would yield more worth-while results. Geographers need to concentrate on particular problems in the spatial structure of our urban society, some of which are sandwiched in the pages of this book. Here are involved questions of criteria, cartographic analysis, and interpretation that cry out for investigation, and are especially fitted to the expertize of the geographer. The defects of this book point to this need, and it is to be hoped that Freeman and his colleagues will apply themselves to it.

R. E. Dickinson

\section{STUDIES IN RHEOLOGY}

Transactions of the Soziety of Rheology

Vol. 2. Edited by R. D. Andrews. Pp. ii +314 . (New York: Interscience Publishers, Inc. ; London: Interscience Publishers, Ltd., 1959.) $64 s$.

\section{Rheology of Disperse Systems}

Proceedings of a Conference organized by the British Society of Rheology, and held at the University College of Swansea, September 1957. Edited by C. C. Mill. Pp. vii +223 . (London and New York: Pergamon Press, 1959.) 60s. net.

$\mathrm{T}$ "HE "Transactions of the Society of Rheology" constitute the proceedings of the annual conferences of the American Society. In 1957, the conference was held in the Textile Research Institute, Princeton, New Jersey. The second of these volumes contains sixteen complete papers and a number of abstracts.

The organizers are to be congratulated on the excellent balance of the programme. It would be tempting to give a heavy preponderance to papers dealing with work in the one or two industries which now employ a high proportion of rheologists; but this has not been done. Theory and practice over a wide field are discussed.

In an introductory paper, A. S. Nowick outlines the career of C. M. Zener, who received the Bingham Medal. Dr. Zener is distinguished in other fields, and it is estimated that his rheological work constitutes less than half his scientific output.

On the more academic side, the subjects treated include work on viscosity-concentration relations in suspensions, relaxation spectra distributions of high polymers, equations for the interconversion of relaxation and retardation spectra and a general study of capillary viscometry.

Papers on applied rheology range over a wide field : the peeling of adhesive tapes, liquid flow in roll nips, creep of snow, mechanical breakdown of bundles of fibres and a fair number of articles on high polymers.

The paper by A. B. Metzner and M. Whitlock is worthy of special mention. Since Osborne Reynolds published his classical work, it has been well known that certain pastes and suspensions become firmer on shearing. All such phenomena have tended to be classed as 'dilatancy', it being assumed that closely packed particles must first pass into open packing before the system can flow, thus producing an increase in volume. This paper confirms what has long been suspected, that the increase in consistency is not necessarily associated with dilatancy at all and often happens at concentrations not corresponding to close packing. The true explanation remains an outstanding problem for rheologists.

'The 'Transactions' will certainly provide an excellent annual survey of the progress of rheology in the United States. It is a pity only that this particular volume has taken so long to appear.

It is difficult to review a collection of eleven papers which, though they are all concerned with dispersed systems (must one omit the grammatically required ' $s$ '?), cover so wide a range of subjects and materials. Yet it is fair to say that, though the standard of the articles differs considerably, the general level is remarkably high.

Prof. J. G. Oldroyd's presidential address deals with the mathematics of 'complicated rheological properties', because, as he points out, simple properties are of little interest to the mathematician. This is fortunate, in view of the complexity of the materials generally studied by practical rheologists.

S. G. Mason and W. Bartok contribute a paper of considerable theoretical interest on the hydrodynamics of suspensions and emulsions, and R. $L$. Whitmore gives some reasons why, on theoretical grounds, suspensions flowing through capillaries fail to obey Poiseuille's fourth-power law, applying his theory to the very important problems of the flow of blood. Since this paper was read, it has stimulated considerable further work. Also N. Casson's and E. Bantoft's contributions, in which they propose a very simple but hitherto unused equation to relate shear stress to rate of shear, has been followed by the publication of descriptions of a number of applications to materials other than varnishes and inks, including blood and molten chocolate. The latter forms the subject of an article by E. H. Steiner, but it was after the conference that he discovered the usefulness of Casson's equation for this material.

A. Jobling and J. E. Roberts, using Weissenberg's deformable membrane technique, describe complex dilatancy effects in certain suspensions which still elude any mechanical explanation and yiold a strong incentive to further study. Other papers deal with high polymers, adhesive sealing compositions ('lutings'), starch-clay mixtures and gaseous fluidized beds. This is clearly a conference which immediately activated further research, thus fulfilling the prime purpose of all such gatherings.

The publishers are to be congratulated on the excellent production of the book. A welcome appendix gives the addresses of all authors and contributors to the discussions. $\quad$ G. W. Soott BLaIR 